will be introduced early in the new session. Viscount Tredegar will introduce it in the House of Lords, while Mr. Linton Thorp has consented to take charge of it when it reaches the House of Commons. Apart from the humanitarian aspect of the steel trap problem, there is another which assumes national importance, inasmuch as it has a vital bearing on agriculture. To agriculturists, the rabbit is a pest, and its extermination would be of great benefit to farmers. Paradoxical as it may seem, the steel trap is beginning to be suspect as an exterminator-and this in districts which have hitherto been wedded to its use. In certain portions of Carmarthenshire and in Pembrokeshire, traps were not used before the War, and rabbits were kept down by other means; since the introduction of the steel trap, these districts are overrun with rabbits.

\section{New Uses for Bone Glue}

THE results of the competition organised by the International Association of Bone Glue Manufacturers ("Epidos"), with the object of extending the uses of bone glue, have recently been announced, and the sum of 30,000 Swiss franes has been distributed among thirty competitors representing ten Continental countries; the fact that this is 10,000 francs in excess of the amount to be distributed under the rules of the competition may be taken as an indication of the high standard of the contributions. It is remarkable that few of the winning memoranda refer to what is usually regarded as the obvious and most common use for glue, namely, as an adhesive. They are, indeed, characterised by the diversity of their interests, and include processes in which glue is used as a stabiliser for colloids (for example, in latex preparations, polishes and ceramic products); as a source of nitrogen in the production of yeast; to enhance resistance (for example, of rubber) to oils and spirits; and as a catalyst, for example, to inhibit the action of acid pickle-liquor. There also appears to be a wide range of uses for glue as an ingredient of plastics, moulding and insulating materials and lacquers, and as a dressing for textiles. Full particulars of each process are obtainable from the General Secretariat of Epidos, 40, Rue du Colisée, Paris. International competitions of this kind suggest a novel method of obtaining technical information which doubtless will prove popular with prospective inventors. In the present instance, the experiment certainly appears to have justified itself, since it is announced that a further competition will be organised in the near future.

\section{B.D.H. Products}

The British Drug Houses Ltd., London, N.1, have issued a handy brochure entitled "B.D.H. Injections for Parenteral Medication". It contains a list of drugs which are commonly given by injection, a brief note of their use and the range of dosage recommended, together with the packings obtainable and their cost. It is stated that the preparation of the solutions is carried out in a specially designed room, provided with double doors and supplied with filtered air at a pressure slightly in excess of atmospheric. The ampoules and bottles used are made of standard alkali-free amber glass and are sterilised after filling by an approved method, the actual process adopted being one which exerts no deleterious action on the medicament. Where containers designed to permit the withdrawal of successive doses on different occasions are employed, a small quantity of antiseptic is added to the solution. "Glucotest Solution" B.D.H. provides a simple and rapid method for determining the amount of sugar in urine. 2 c.c. of the solution is boiled in a test tube with a small amount of Glucotest powder to prevent bumping and the urine is added drop by drop from a pipette. The addition of urine is continued until the blue colour of the liquid has completely disappeared and a white or yellow colour free from any suggestion of green remains. The amount of glucose in the urine is inversely proportional to the number of drops required, and is ascertained directly from a table supplied with the solution.

\section{Cosmic Radiation}

No. 4 of the Annals of the Observatory of Lund, 1934, is devoted to a memoir in English, entitled "Cosmic Ultra-Radiation in Northern Sweden (an Academical Dissertation)" by Axel Corlin. It is an admirably printed quarto volume containing 113 pages of text and 80 pages of tables and bibliography. The author made measurements of the cosmic radiation in the far north of Sweden, using a Kolhörster apparatus in 1929-30 and a Steinke apparatus in 1932-34. A careful study is made of the relation between the radiation measured, and the air pressure, air temperature and humidity, using the method of multiple correlation. No direct influence of air temperature and humidity was found. Likewise no positive relation was established, after exhaustive investigation, between the cosmic radiation and magnetic storms and auroræ. The transition effect in iron was observed in a lake near Abisko, with results similar to those found by Steinke at Königsberg. The ionisation by cosmic radiation was measured in the Kirunavaara iron mine, and was detected down to 700 metres water-equivalent. The volume contains two chapters of great general interest, one giving a historical summary of the experimental and theoretical development of cosmic ray investigation, while the other discusses the origin of the radiation, the present situation of this problem being described as "quite desperate".

\section{Measurement of Geological Time}

IN 1931, H. V. Ellsworth analysed a specimen of uraninite from Manitoba and obtained a lead-ratio with the surprisingly high value of $0 \cdot 260$, corresponding to an age of about 1,750 million years. Although Ellsworth gave adequate evidence that the mineral was of first class quality, there has naturally been some hesitation in accepting this great extension of geological time. Confirmation of the most convincing kind is now, however, forthcoming. It is announced by Prof. A. C. Lane through Science Service, Washington, D.C., that Miss Edith Kroupa (working in the laboratory of Dr. F. Hecht in Vienna) has 